\title{
Time-Frequency Transforms for Classification of Power Quality Disturbances
}

\author{
Alejandro Rodríguez, Jose A. Aguado, Jose J. López, \\ Francisco Martín, Francisco Muñoz and Jose E. Ruiz \\ University of Malaga \\ Spain
}

\section{Introduction}

The usual operations on the distribution network such as switching loads and circuits, the proliferation of power electronic equipment and non-linear loads and the distributed generation with renewable energy are several of the most common causes that are leading to an increasing polluted power system in terms of voltage signal distortion.

One way of improving the power quality (PQ) parameters consists of analyzing these disturbances efficiently and understanding them deeply (Dugan, 2000) and PQ monitoring is one major task in order to achieve it. PQ monitoring is not an easy task usually involving sophisticated hardware instrumentation and software packages. Many recent approaches in PQ monitoring try to achieve it through the automated classification of different disturbances.

The different approaches in this field lead their efforts in two directions, the main parts that form an automated classification as depicted in Fig. 1. The first make focus to obtain a

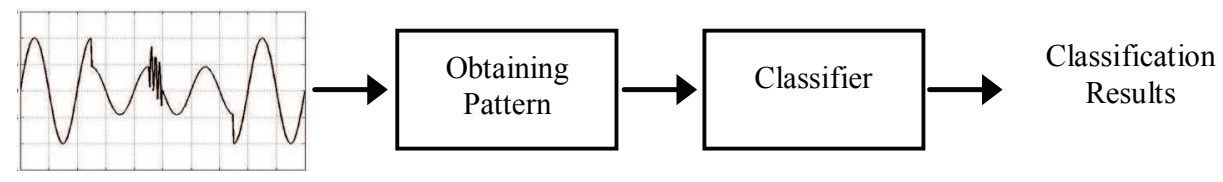

Fig. 1. Automated classification scheme

suitable pattern that allow distinguish clearly each disturbance, by the use of signal processing tool. Among existing signal processing tools the Fourier Transform (FT) results inadequate for analysis of non-stationary events and Short-Time Fourier Transform (STFT) although improves this drawback, it does not achieve good resolution in both time and frequency. Nowadays time-frequency transforms are used to get feature extraction, such as Wavelet transform (WT) (Santoso, 1994) and S-transform (ST) (Dash, 2003). WT extracts information from the signal in time and frequency domains, simultaneously, and provide greater resolution in time for high frequency components of a signal and greater resolution in frequency for the low frequency components of a signal. The ST can conceptually be 
interpreted as a hybrid of STFT and WT. It uses variable window length and, by using the FT kernel, it can preserve phase information during the decomposition (Stockwell et al., 1996). The frequency-dependent window produces higher frequency resolution at lower frequencies, while at higher frequencies, sharper time localization can be achieved.

The second approach is oriented to use a classifier able to assign each disturbance correctly in its class, so the most of the artificial intelligent techniques have been combined with WT or ST, as Artificial Neural Networks (ANN), Decision Tree Fuzzy Logic, Hidden Markov Model, Support Vector Machines, etc.

In this chapter two different classification systems have been developed, using the WT and ST for pattern extraction, and an ANN as classifier algorithm.

The features obtained from WT are not completely distinctive and it is necessary to add features that give clear information about the signal magnitude. The real mean squared (RMS) value of the voltage signal have been obtained to achieve it.

On the other hand, the features obtained from ST analysis are sufficient to achieve a pattern that can properly classify the disturbances. In order to increase accuracy, simplicity and reliability, this chapter proposes a reduced and simple set of features extracted from the ST. Even in the presence of complex disturbances with different levels of noise, these features characterize the signals in a suitable way.

This chapter is organized as follows. In the second section the time-frequency transforms used in power quality are presented, particularly the WT and ST, and the obtaining pattern using these two transforms. A brief description of ANN used as classifier algorithm used in this chapter is given in section three. In section fourth the classification results obtained using the resulting classification system are presented. These systems are checked by signals obtained from electric power simulation in section fifth. Finally, conclusions are presented.

\section{Time-frequency transforms and obtaining pattern}

\subsection{Fourier transform}

The most used classical signal processing is the FT. This transform represents a signal as a sum of sinusoidal terms of different frequencies, named the frequency spectrum. This technique is suitable for stationary signals, but it is not efficient when the signal contents short-term transient disturbances.

In order to solve this drawback, a technique based on the FT is applied to short time intervals. This method is known as "Short Time Fourier Transform" (STFT), and consists of analyzing by the FT a sliding window of the signal. It is not possible reaching a good resolution in time and frequency simultaneously, and therefore it is necessary to adopt a compromise solution between the frequency and time resolutions.

The STFT is obtained by choosing a sliding window (short time interval) where the FT is applied. For narrow windows (or short time intervals) a good time resolution is obtained, suitable for short-term transients; on the other side a relative wide window enables a good frequency resolution but gives inaccurate time resolution.

Therefore, the problem lies in the fact that the window width is a parameter that must be fixed before analyzing the signal, before knowing what resolution is more suitable. At last, the total number of operations for computing STFT is $N \cdot \log N$.

\subsection{Wavelet transform}

Wavelet analysis is a powerful tool widely used in many scientific applications, especially in transient, non stationary, or time-varying situations. 
A wavelet can be considered as a small wave which has its energy concentrated in time, and fits certain mathematic properties (Burrus et al., 1998). Fig. 2 shows three examples of wavelet.

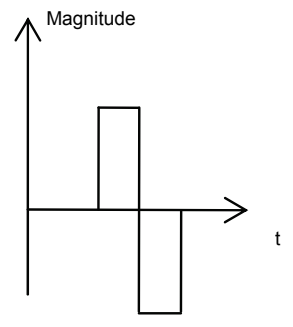

Haar

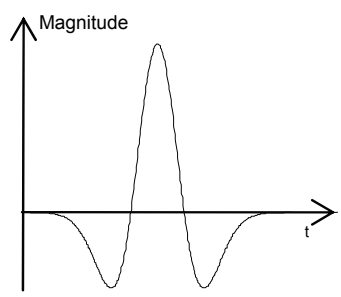

Mexican hat

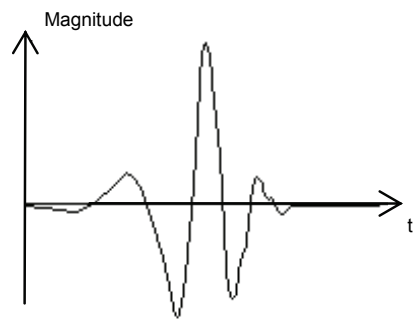

Daubechies 4

Fig. 3. Three examples of wavelet

The mother wavelet $\psi(t)$ can be scaled and translated in the time, generating a family of functions named the wavelet expansion set (Wavelet system):

$$
\psi_{j, k}(t)=2^{j / 2} \psi\left(2^{j} t-k\right)
$$

A function $f(t)$ can be expressed as a linear decomposition of this wavelet system as follows:

$$
f(t)=\sum c_{j, k} \psi_{j, k}(t), j, k \in Z
$$

where $j$ and $k$ are integer indices, $c_{j, k}$ are real coefficients, and $\psi_{j, k}(t)$ is the expansion set. The set of expansion coefficients $c_{j, k}$ is called the wavelet spectrum of the function $f(t)$.

If the wavelet expansion system is orthogonal, then:

$$
j \neq l \text { or } k \neq m \Rightarrow\left\langle\psi_{j, k}(t), \psi_{l, m}(t)\right\rangle=0
$$

where $<>$ denotes the inner product defined as:

$$
\left\langle\psi_{j, k}(t), \psi_{l, m}(t)\right\rangle=\int \psi_{j, k}(t) \cdot \psi_{l, m}(t) d t
$$

When the expansion wavelet system is orthogonal the Wavelet spectrum can be computed as follows:

$$
c_{j, k}=\int \psi_{j, k}(t) \cdot f(t) \cdot d t
$$

The index $j$ is related with the frequency and the index $k$ with time.

In practical applications a minimum frequency have to be established.

For a mother wavelet a basic scaling function $\varphi(t)$ is defined. A set of scaling functions is defined in terms of integer translates of $\varphi(t)$ by

$$
\varphi_{k}(t)=\varphi(t-k)
$$

A two-dimensional family of scaling functions can be defined by scaling and translation by 


$$
\varphi_{j, k}(t)=2^{2 / j} \varphi\left(2^{j} t-k\right)
$$

Let consider the minimum frequency is the corresponding to the value $j=J_{0}$.

The equation (2) becomes to

$$
f(t)=\sum_{k} \sum_{j} c_{j, k} \Psi_{j, k}(t)=\sum_{k} a_{J_{0}, k} \varphi_{J_{0}, k}(t)+\sum_{j=J_{0}}^{\infty} \sum_{k} d_{j, k} \Psi_{j, k}(t), \quad t \in R
$$

where $J_{0}$ is an integer. Equation (4) is a linear combination of wavelet coefficients, $\left(a_{J o, k}, d_{j, k}\right)$, a set of functions $\varphi_{I o, k}(t)$, called scaling functions and $\Psi_{j, k}(t)$, called wavelet functions. Coefficients $a_{J o, k}$ and $d_{j, k}$ are the Discrete Wavelet Transform $(D W T)$ of $f(t)$, and can be calculated as:

$$
a_{J_{0}, k}=\left\langle f(t), \varphi_{J_{0}, k}(t)\right\rangle \quad d_{j, k}=\left\langle f(t), \Psi_{j, k}(t)\right\rangle
$$

Equation (4) can be truncated for $j=J-1$, obtaining:

$$
f(t)=\sum_{k=0}^{2^{J 0}-1} a_{J_{0}, k} \varphi_{J_{0}, k}(t)+\sum_{j=J_{0}}^{J-1} \sum_{k=0}^{2^{j}-1} d_{j, k} \Psi_{j, k}(t), \quad t \in R
$$

The first summation in (6) is a broad representation of $f(t)$ that has been expressed as a linear combination of $2^{J_{0}}$ translations of the scaling function, $\varphi_{I 0,0}$. The second summation contains the details of $\mathrm{f}(t)$. For each level $j$, a linear combination of $2^{j}$ translations of the wavelet function, $\psi_{j 0}$, are added to obtain a more accurate approximation of $f(t)$.

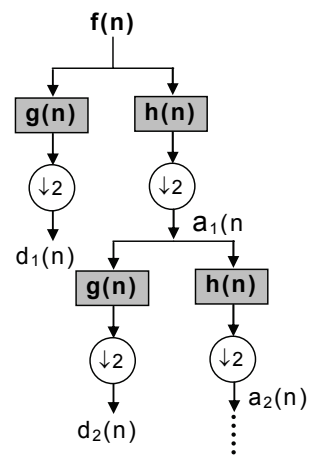

Fig. 2. Mallat algorithm schematics

The Mallat algorithm (Mallat, 1999) has been used in the practical implementation of DWT. The DWT acts as two FIR (Finite Impulse Response) quadrature filters defined by two sequences $h(n)$ and $g(n) . h(n)$ is a high frequency filter and $g(n)$ is a low frequency filter. Both filter have the same cut frequency $f_{N} / 2$, where $f_{N}$ is the Nyquist frequency. Therefore the function $f(n)$ is split in two parts, the high frequency part $d_{1}$ that contains the higher octave and is called detail function, and the low frequency part $a_{1}$, that contains the frequencies lower than $f_{N} / 2$, and is called smoothed function. Decimation by 2 is done for eliminating redundant information. The algorithm is iterated for $a_{1}$, obtaining a second level detail function $d_{2}$ and a second level smoothed function $a_{2}$, that is again splitted, obtaining a series of detail and broad functions. The total number of operations for computing DWT is N. 
The original function $f(n)$ is split into a series of detail functions $d_{1}, d_{2}, \ldots, d_{k}$, and a smoothed function $a_{k}$, that correspond to the frequencies:

$d_{1}: f_{N}-f_{N} / 2 ; d_{2}: f_{N} / 2-f_{N} / 4 ; \ldots . d_{k}: f_{N} / 2^{n}-f_{N} / 2^{k+1} ;$ and $a_{n}$ contains the frequencies lower than $f_{N} / 2^{k+1}$.

Fig. 3 and Fig. 4 show an example of the application of the Mallat multi-resolution algorithm to the wavelet spectrum computation of a signal with a sag to $40 \%$.

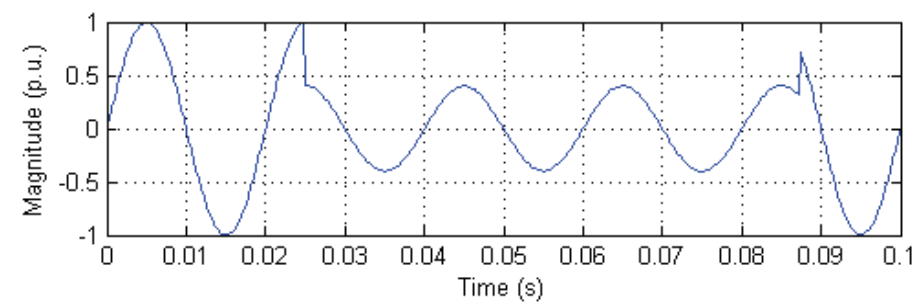

Fig. 3.Voltage sag to $40 \%$ and 400 samples length

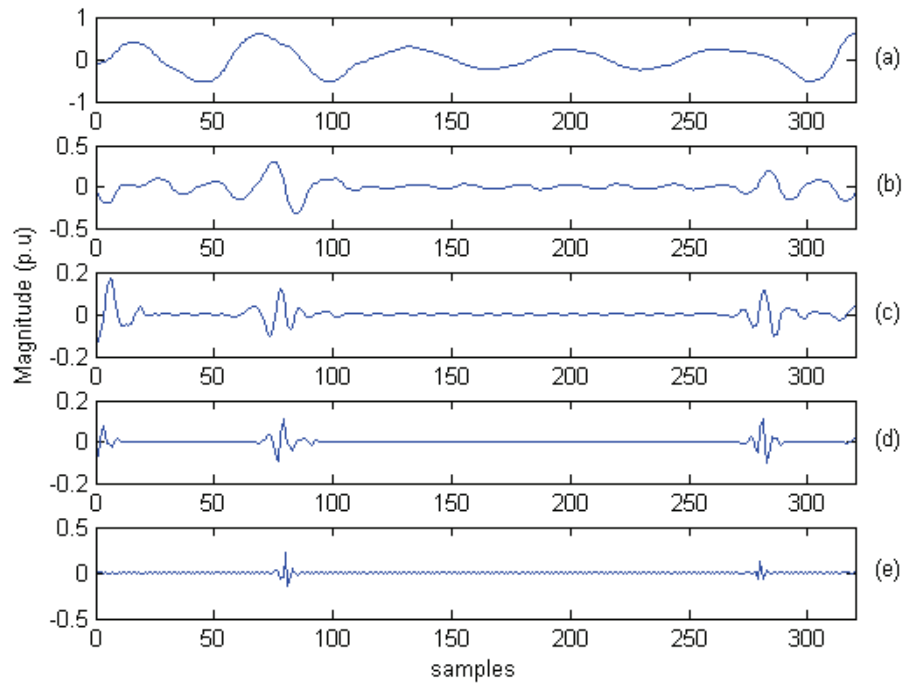

Fig. 4. Voltage sag detail levels wavelet analysis, from d1 to d5, (a) to (e), respectively

It is important to note that the wavelet is not a single specified function but a framework within which can design different wavelets. In this work, Daubechies 3 (db3) has been used as wavelet mother, performing 5 levels of decomposition. A voltage sag signal is shown in Fig. 3, and its wavelet transform decomposition, using db3, can be seen in Fig. 4.

\subsection{Wavelet based pattern}

The pattern used in this work is based on Parseval's Theorem. This Theorem states that the energy of a signal $f(t)$ remains the same whether it is computed in a signal domain (time) or in a transform domain (frequency) as follows: 


$$
E_{\text {signal }}=\frac{1}{T} \int_{0}^{T}|f(t)|^{2} d t=\sum_{n=0}^{N}|F[n]|^{2}
$$

where $T$ and $N$ are the time period and the length of the signal, respectively, and $F(n)$ is the Fourier transform of the signal. In the case of the DWT, the signal is decomposed in terms of bands of frequencies, thus the energy of a signal can be given as:

$$
E_{D W T}=\frac{1}{T} \int_{0}^{T}|f(t)|^{2} d t=\sum_{l=-\infty}^{\infty}|a(l)|^{2}+\sum_{j=0}^{\infty} \sum_{k=-\infty}^{\infty}\left|d_{j}(k)\right|^{2}
$$

with the energy in the expansion domain partitioned in time by $\mathrm{k}$ and scaled by $\mathrm{j}$.

The sampling frequency of the signals is $3.2 \mathrm{kHz}$, which is equal to $64 f_{1}$ where $f_{1}$ is the power system frequency $(50 \mathrm{~Hz}$ in Europe). The wavelet spectrum contains the total information of the original waveform as shown in Table 1.

\begin{tabular}{|c|c|}
\hline DWT coefficients & Frequency band \\
\hline $\mathrm{d}_{1}$ & $32 \mathrm{f}_{1} \div 16 \mathrm{f}_{1}$ \\
\hline $\mathrm{d}_{2}$ & $16 \mathrm{f}_{1} \div 8 \mathrm{f}_{1}$ \\
\hline $\mathrm{d}_{3}$ & $8 \mathrm{f}_{1} \div 4 \mathrm{f}_{1}$ \\
\hline $\mathrm{d}_{4}$ & $4 \mathrm{f}_{1} \div 2 \mathrm{f}_{1}$ \\
\hline $\mathrm{d}_{5}$ & $2 \mathrm{f}_{1} \div \mathrm{f}_{1}$ \\
\hline
\end{tabular}

Table 1. Frequency band information contained in the wavelet spectrum.

In certain signals an energy-based pattern is not completely discriminatory because the energy of the magnitude disturbance depends on the depth of the disturbance and its duration in time.

The spectra of normal signals, sags and swells do not contain energy in the bands up to the fundamental frequency, as these signals only differ in the magnitude of the fundamental frequency component, and present very few energy in the high frequencies.

Therefore, it is necessary to provide a feature based on the magnitude of the signal. The RMS value is a widely accepted tool that provides information about how much the magnitude of the voltage changes. It is a fast and simple algorithm (13) that requires very few computational resources.

$$
V_{R M S}=\sqrt{\frac{1}{N} \sum_{n=1}^{N}(f(n))^{2}}
$$

where $f(n)$ is the signal of length $N$.

The digital measurement instruments perform the computation of this parameter from instantaneous values of the samples, choosing a temporary window depending on the frequency of the steady state signal. If the RMS values are updated when a new sample is acquired, the method is called RMS continuum. If the RMS values are updated at a certain interval, usually half cycle, then it is called RMS (1/2). In this work the RMS $(1 / 2)$ has beencomputed, selecting the maximum and minimum values. These values provide a feature based on the signal magnitude, as shown in Table 3. 


\begin{tabular}{|l|l|l|}
\hline & \multicolumn{2}{|l|}{ RMS voltage values } \\
\hline Signal & Max & Min \\
\hline Perfect signal & 1.001 & 0.999 \\
\hline Normal to 0.91 in 4.5 cycles & 1.001 & 0.909 \\
\hline Sag to 0.8 in 1 cycle & 1.001 & 0.799 \\
\hline
\end{tabular}

Table 3. Maximum and minimum RMS voltage value for different signals

Therefore the pattern is made up in two stages. The first part has five values, the energy of the signal in each frequency band of wavelet decomposition (feature extraction). These values have been normalized with reference to the values obtained from an ideal sinusoidal signal. The second part of the pattern is formed for two values the maximum and minimum RMS value calculated directly (feature selection) from each signal.

\subsection{S-transform}

The S-Transform (Stockwell et al., 1996) is a time-frequency transform generated by the combination of WT and STFT. The S-transform $s(\tau, f)$ of the signal $x(t)$ is defined as follows:

$$
S(\tau, f)=\int_{-\infty}^{\infty} X(t) g_{f}(\tau-t) \exp (-j 2 \pi f t) d t
$$

where

$$
g_{f}(\tau-t)=\frac{|f|}{\sqrt{2 \pi}} \exp \left(\frac{-f^{2}(\tau-t)^{2}}{2}\right)
$$

$g_{f}(\tau-t)$ is the Gaussian window function, $\tau$ is a shift parameter for adjusting the position in the time axis and $f$ is the scale parameter.

$X(f)$ is defined as the Fourier transform of $x(t)$. The relationship between the S-transform and the Fourier transform is:

$$
X(f)=\int_{-\infty}^{\infty} S(\tau, f) d x
$$

The discrete ST is defined by:

$$
S\left(\frac{n}{N T}, j T\right)=\sum_{m=0}^{N-1} X\left(\frac{m+n}{N T}\right) \cdot \exp \left(-\frac{2 \pi^{2} m^{2} k^{2}}{n^{2}}\right) \exp \left(\frac{i 2 \pi n j}{N}\right)
$$

In order to obtain the ST, the FFT of the original signal is computed [17]. The total number of operations for computing ST is $N \cdot(N+\log N)$.

The multi-resolution ST is a complex matrix whose rows and columns values are frequency and are time values, respectively. Each column represents the local spectrum in time. Frequency-time contours having the same amplitude spectrum are also obtained. This information is used to detect and characterize power disturbance events.

A mesh-dimensional output of the ST yields frequency-time, amplitude-time and frequencyamplitude plots. Examples of multi-resolution ST analysis for signals containing harmonics 
and oscillatory transients are presented in Fig. 2 and 3, respectively. Each figure plots the disturbance signal (a), the time-frequency contours (b), and 3-D mesh giving amplitude, frequency and time plots (c).
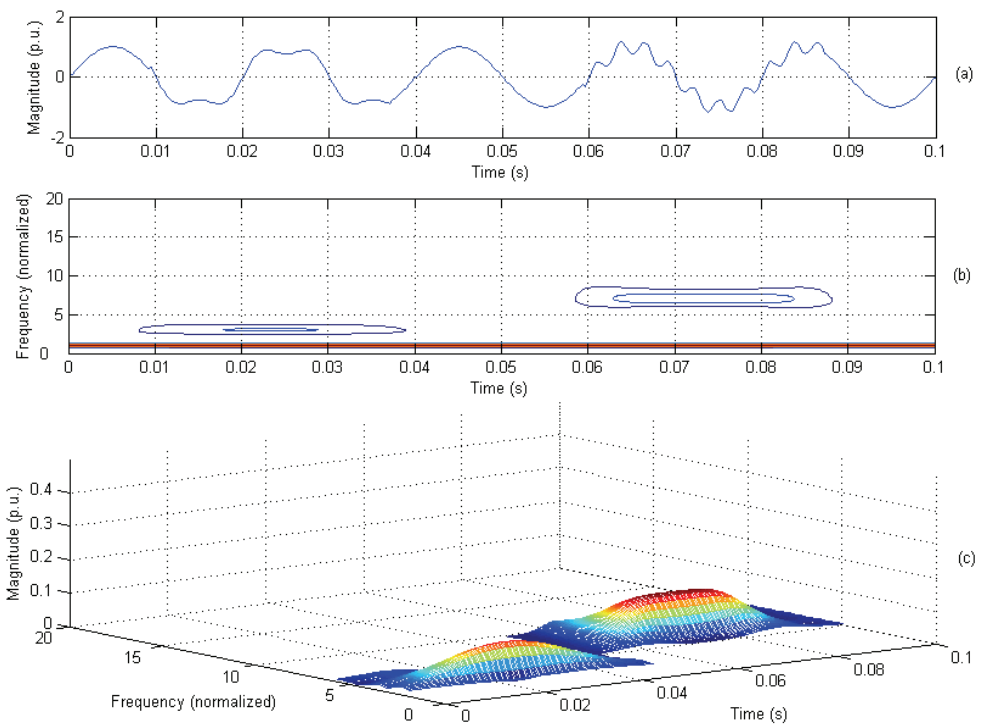

Fig. 2. Signal with 3rd and 7th harmonic content (a). S-transform Time-Frequency contours (b). 3D mesh Time-Frequency-Amplitude (c).
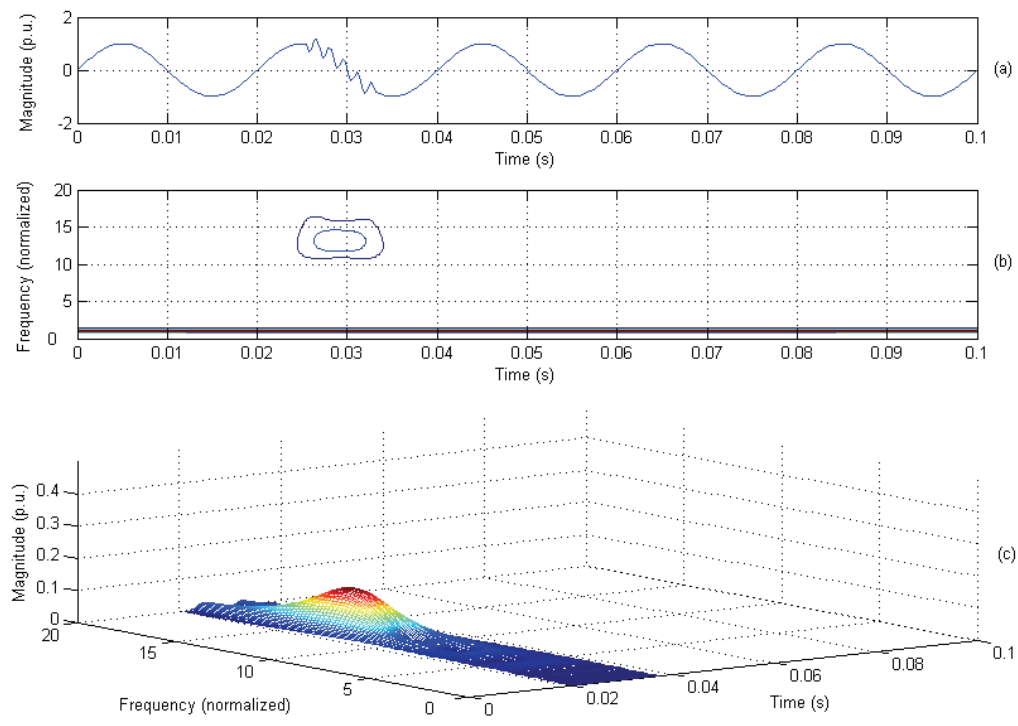

Fig. 3. Oscillatory transient (a). S-transform Time-Frequency contours (b). 3D mesh TimeFrequency-Amplitude (c). 


\subsection{S-transform based pattern}

An efficient pattern can be defined from observation of ST contours. Below some examples of signals with different disturbances are analyzed in order to illustrate the pattern proposed in this approach.

A sinusoidal signal without any disturbance is depicted in Figure 4 (a). The fundamental frequency contour (b) shows a horizontal line. Other frequency contours (c), (d) and (e), corresponding to 150,250 and $700 \mathrm{~Hz}$ show the non-existence of these frequencies in the signal.
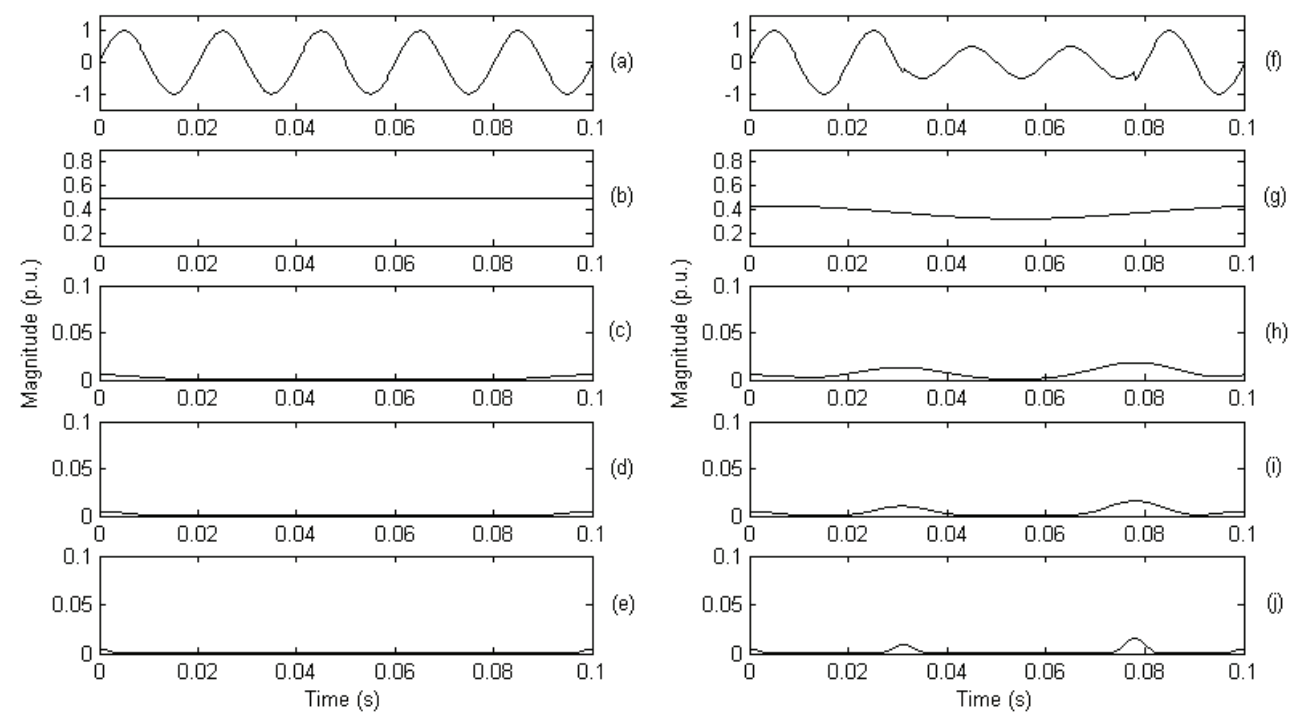

Fig. 4. Pure sinusoidal signal and $50 \mathrm{~Hz}, 150 \mathrm{~Hz}, 250 \mathrm{~Hz}$ and $700 \mathrm{~Hz}$ ST contours (a) to (e), and voltage sag, $50 \mathrm{~Hz}, 150 \mathrm{~Hz}, 250 \mathrm{~Hz}$ and $700 \mathrm{~Hz}$ ST contours from (f) to (j), respectively.

A sinusoidal signal with voltage sag and several frequency contours are depicted in Fig. 4 (f-j). The $50 \mathrm{~Hz}$ contour $(\mathrm{g})$ is observed to decrease its value during the voltage sag. In the case of an interruption (not shown), a similar behaviour can be observed, but with a deeper diminution. The frequency contours corresponding to other frequencies (h-j) present a low energy value at the beginning and end of the sag.

An oscillatory transient disturbance is depicted in Figure 6 (a) and the $150 \mathrm{~Hz}, 250 \mathrm{~Hz}, 350$ $\mathrm{Hz}$ and $700 \mathrm{~Hz}$ contours in (b) to (e), respectively. A big amount of energy can be noted in the contour corresponding to the frequency present in the transient signal, $700 \mathrm{~Hz}$ in this particular case.

Figure 6 (f) depicts a sinusoidal signal with a third and a fifth harmonic simultaneously, and several frequency contours. It clearly shows a big amount of energy in the contours corresponding to the frequencies present in the signal, 150 and $250 \mathrm{~Hz}$, Fig. 6 (h-i).

The examples shown above illustrate the way of taking advantage of this particularity of the ST in order to search for specific frequency disturbances such as any order harmonics or particular oscillatory transients. The results shown above have been taken into account in the election of the characteristic features. 

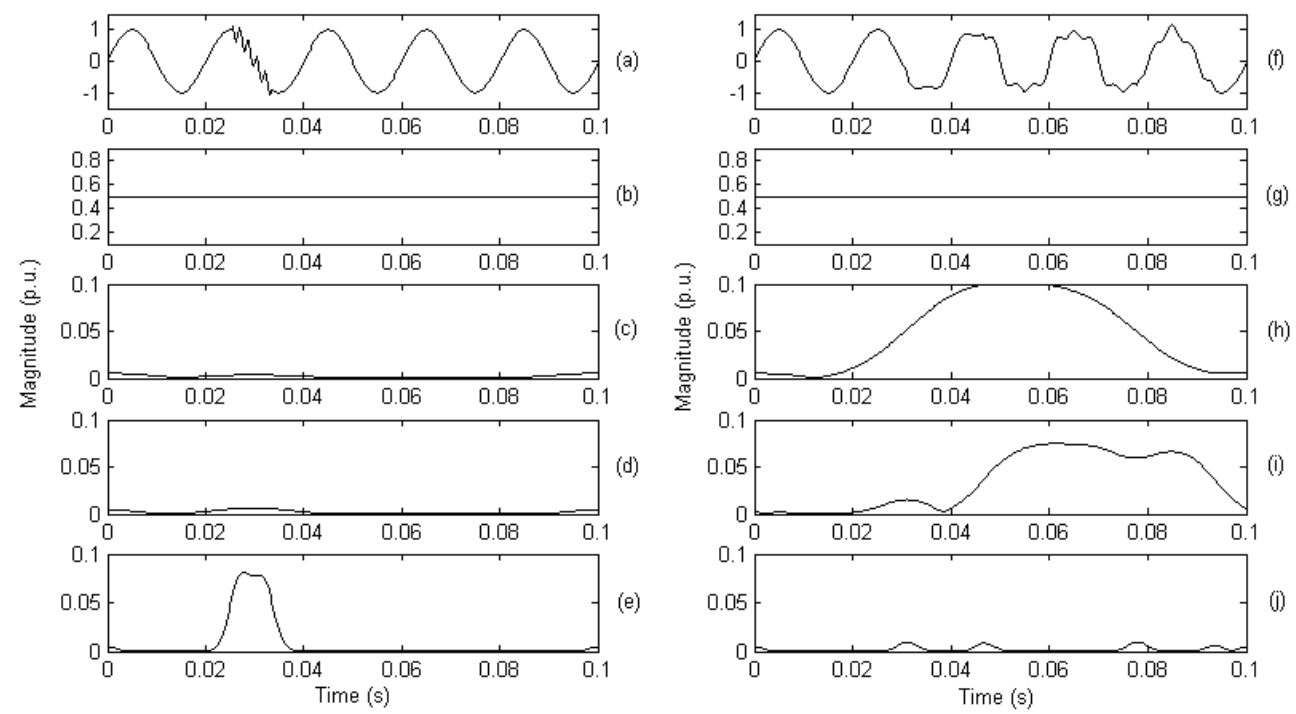

Fig. 4. Oscillatory transient, $50 \mathrm{~Hz}, 150 \mathrm{~Hz}, 250 \mathrm{~Hz}$ and $700 \mathrm{~Hz}$ ST contours (a) to (e), respectively, and signal with third and fifth harmonic component, $50 \mathrm{~Hz}, 150 \mathrm{~Hz}, 250 \mathrm{~Hz}$ and $700 \mathrm{~Hz}$ ST contours from (f) to (j), respectively.

The fundamental frequency contour has proven to contain valuable information about sags, swells and interruptions. Hence, the mean value of the $50 \mathrm{~Hz}$ contour has been taken as a distinctive feature. But this value does not clearly discriminate among sags and interruptions, and therefore the minimum value of the $50 \mathrm{~Hz}$ contour, which gives an idea of the severity of the disturbance, has been taken as the second feature.

In order to discriminate disturbances with presence of harmonics, the energy of the third, fifth and seventh harmonic $(150 \mathrm{~Hz}, 250 \mathrm{~Hz}$ and $350 \mathrm{~Hz})$ contours are used as distinctive features. This approach has been restricted to these frequencies although it could be extended to other harmonics within the Nyquist condition.

The sum of the energies from $600 \mathrm{~Hz}$ to $1600 \mathrm{~Hz}$ (Nyquist frequency) has also been taken as another characteristic feature. A high value of this energy gives information related to high frequency transient events.

A summary of the distinctive features used in ST based pattern is listed below:

F1: Mean of the fundamental frequency contour $(50 \mathrm{~Hz})$

F2: Minimum of the fundamental frequency contour

F3: Energy of the 3rd harmonic contour $(150 \mathrm{~Hz})$

F4: Energy of the 5th harmonic contour $(250 \mathrm{~Hz})$

F5: Energy of the 7 th harmonic contour $(350 \mathrm{~Hz})$

F6: Sum of energy from 600 to $1600 \mathrm{~Hz}$ contours

\section{Artificial neural network}

As algorithm in the automated classification scheme has been selected ANN. Neural networks have emerged as an important tool for classification, and have been successfully applied to a variety of real world classification tasks in industry, business and science 
(Widrow et al., 1994). Applications include classification of power quality disturbances (Borras et al. 2001).

An ANN is composed of very simple elements operating in parallel. These elements are inspired by biological nervous systems. As in nature, the network function is determined largely by the connections between elements. An ANN can be trained to perform a particular function by adjusting the values of the connections between elements. The ANNs can approximate any function with arbitrary accuracy, and they are nonlinear models, which makes them flexible in modeling real world complex relationships. Moreover ANNs are data driven self-adaptive methods in that they can adjust themselves to the data without any explicit specification of functional or distributional form for the underlying model (Zhang, 2000). In this chapter two different ANNs have been used, backpropagation and probabilistic.

\subsection{Backpropagation}

Feedforward backpropagation (BP) is a gradient descent algorithm, in which the network weights are moved along the negative of the gradient of the performance function. The term $\mathrm{BP}$ refers to the manner in which the performance function is propagated from the output to backward, and feedforward to the direction of the different connection between elements.

Once the network weights and biases have been initialized, the network is ready for training. The training process requires a set of examples of proper network behaviour, network inputs and target outputs. During training the weights and biases of the network are iteratively adjusted to minimize the network performance function. The default performance function for feedforward networks is mean square error, (i.e. the average squared error between the network outputs and the target outputs).

The BP has been set with two layers, one of them is a hidden layer. This structure can uniformly approximate any continuous function (Cybenko, 1989). The number of nodes in the hidden layer has been chosen as a function of number of inputs (Hecht-Nielsen, 1989), i.e. the number of pattern features, according to the expression $(2 n+1)$, where $n$ is the number of inputs. So for each pattern used, the hidden layer has different number of nodes, and the output layer only has one. The transfer functions for the hidden and output layer are tansigmoidal and lineal, respectively. The learning ratio has been 0.1 , the epoch 3500 and the training algorithm Levenberg-Marquadt.

\subsection{Probabilistic}

The basic principle of probabilistic neural network (PNN) is implemented using the probabilistic model, such as Bayesian classifiers (Specht, 1990). The training examples are classified according to their values of probabilistic density function. When an input is presented, the first layer computes distances from the input vector to the training input vectors, and produces a vector whose elements indicate how close the input is to a training input. The second layer sums these contributions for each class of inputs to produce a vector of probabilities as output. Finally, a complete transfer function on the output of the second layer picks the maximum of these probabilities (Mishra et al., 2008). The most obvious advantage of this network is that training is trivial and instantaneous.

\section{Classification results}

Once the algorithm classifier is selected it has to be trained. At this stage, the classification algorithm is adjusted so that the function that assigns data to its corresponding class has the 
lowest possible error based on a particular set of training data. In this way the system learns to match the output of the function with the tag or class for each data.

Once the classifier has been adjusted, the results are validated with a different data set, in order to check the effectiveness of the whole system.

The data set consists 6000 signals, where $10 \%$ are used to verify the effectiveness of the network, so 900 and 100 for each class, have been used for training and verification stage, respectively.

In this section the most usual power quality disturbances have been considered, sags, interruptions (int), swells, oscillatory transients (OT), harmonics (harm) and signals considered as normal voltage (normal). These signals are generated following mathematical models presented in (Gargoom et al., 2008), simulated in Matlab [Matlab, 2000] with a fundamental frequency of $50 \mathrm{~Hz}$ and a virtual voltage of $1 \mathrm{~V}$. The signals have a five cycles length $(100 \mathrm{~ms})$ and the sampling frequency is $3.2 \mathrm{kHz}$.

\subsection{Classification using WT based pattern and ANN}

The resulting classification system using WT based pattern is presented in Fig. 8. It can be observed that the obtained pattern is composed of a feature selection (RMS value) and feature extraction. Selection chooses distinctive features from a set of candidates, while extraction utilizes some transformations, WT in this case, to generate useful and novel features from the original ones (Xu \& Wunsch, 2005).

The classification results obtained are presented in Table 4 and 5, using BP and PNN as algorithm classifier, respectively.

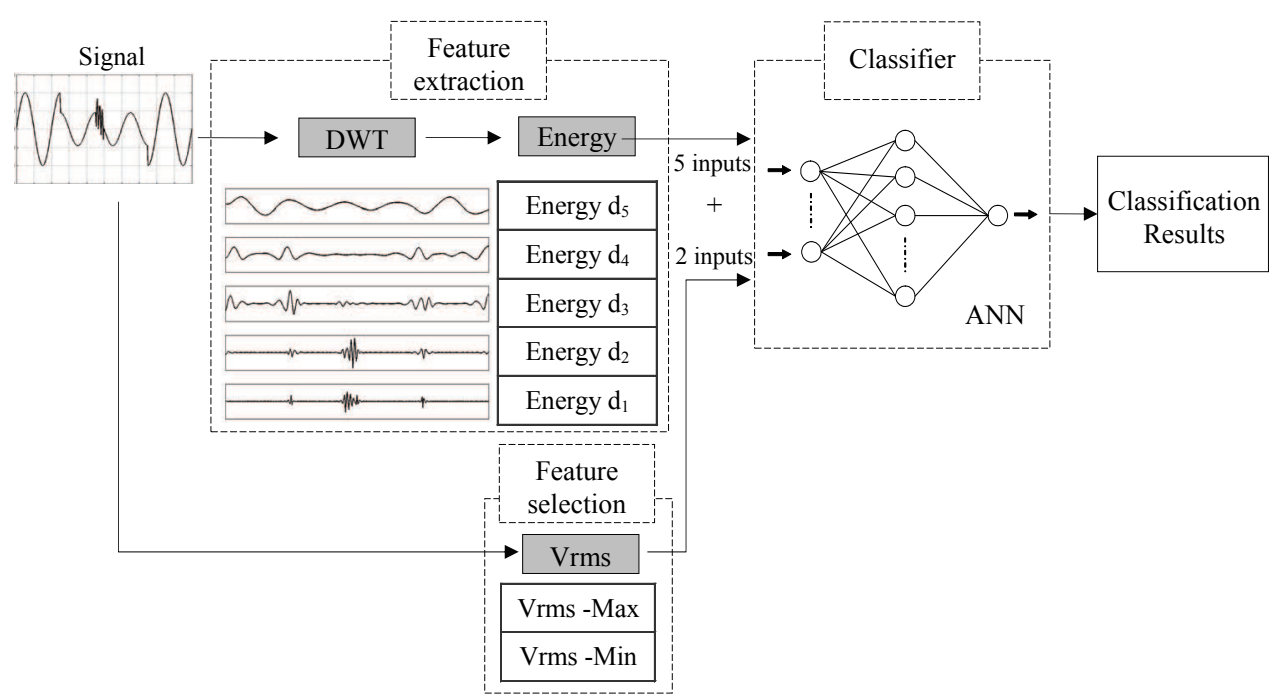

Fig. 8. Resulting classification system using wavelet based pattern and ANN

As can be observed the classification results are slightly better with BP than PNN except with high level of noise, 20dB. For disturbances, the worst results are obtained for sag and interruptions. 


\begin{tabular}{|c|c|c|c|c|}
\hline & No noise & $\mathbf{4 0} \mathbf{d B}$ & $\mathbf{3 0} \mathbf{~ d B}$ & $\mathbf{2 0} \mathbf{d B}$ \\
\hline Normal & 0.97 & 0.99 & 0.97 & 0.8 \\
\hline Sag & 1 & 1 & 0.95 & 0.91 \\
\hline Int & 0.99 & 0.97 & 1 & 0.94 \\
\hline Swell & 1 & 1 & 0.99 & 0.98 \\
\hline OT & 0.9 & 0.96 & 1 & 1 \\
\hline Harm & 1 & 1 & 1 & 0.86 \\
\hline Total & $\mathbf{0 . 9 7 7}$ & $\mathbf{0 . 9 8 7}$ & $\mathbf{0 . 9 8 5}$ & $\mathbf{0 . 9 1 5}$ \\
\hline
\end{tabular}

Table 4. Classification results using WT based pattern and BP

\begin{tabular}{|c|c|c|c|c|}
\hline & No noise & $\mathbf{4 0} \mathbf{d B}$ & $\mathbf{3 0} \mathbf{~ d B}$ & $\mathbf{2 0} \mathbf{d B}$ \\
\hline Normal & 1 & 0.99 & 0.99 & 0.99 \\
\hline Sag & 0.91 & 0.93 & 0.96 & 0.96 \\
\hline Int & 0.74 & 0.82 & 0.92 & 0.92 \\
\hline Swell & 0.96 & 1 & 1 & 1 \\
\hline OT & 1 & 0.96 & 0.99 & 0.99 \\
\hline Harm & 1 & 1 & 0.99 & 0.99 \\
\hline Total & $\mathbf{0 . 9 3 5}$ & $\mathbf{0 . 9 5 0}$ & $\mathbf{0 . 9 7 5}$ & $\mathbf{0 . 9 4 3}$ \\
\hline
\end{tabular}

Table 5. Classification results using WT based pattern and PNN

\subsection{Classification using ST based pattern and ANN}

The resulting classification system using ST based pattern is presented in Fig. 9. The classification results are presented in Table 6 and 7, using BP and PNN as algorithm classifier, respectively.

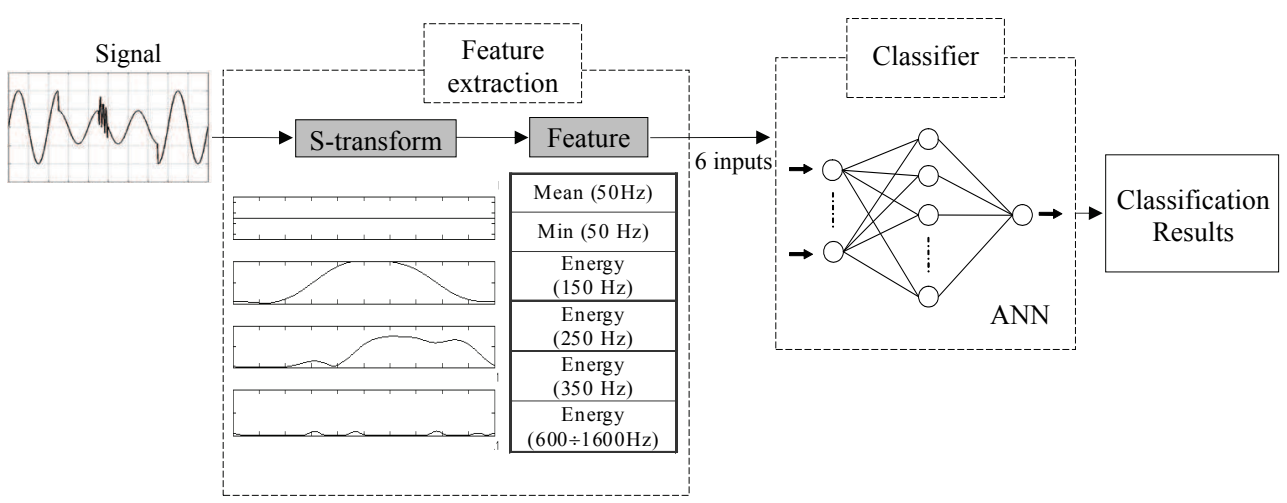

Fig. 9. Resulting classification system using ST based pattern and ANN 


\begin{tabular}{|c|c|c|c|c|}
\hline & No noise & $\mathbf{4 0} \mathbf{~ d B}$ & $\mathbf{3 0} \mathbf{~ d B}$ & $\mathbf{2 0 ~ d B}$ \\
\hline Normal & 1 & 0.98 & 0.99 & 0.94 \\
\hline Sag & 0.99 & 0.96 & 0.99 & 0.93 \\
\hline Int & 0.79 & 0.98 & 1 & 0.99 \\
\hline Swell & 0.97 & 0.95 & 0.96 & 0.99 \\
\hline OT & 1 & 1 & 1 & 0.99 \\
\hline Harm & 1 & 1 & 1 & 1 \\
\hline Total & $\mathbf{0 . 9 5 8}$ & $\mathbf{0 . 9 7 8}$ & $\mathbf{0 . 9 9 0}$ & $\mathbf{0 . 9 7 3}$ \\
\hline
\end{tabular}

Table 6. Classification results using ST based pattern and BP

\begin{tabular}{|c|c|c|c|c|}
\hline & No noise & $\mathbf{4 0} \mathbf{~ d B}$ & $\mathbf{3 0} \mathbf{d B}$ & $\mathbf{2 0} \mathbf{~ d B}$ \\
\hline Normal & 1 & 1 & 1 & 0.99 \\
\hline Sag & 0.79 & 0.65 & 0.57 & 0.62 \\
\hline Int & 0.61 & 0.73 & 0.69 & 0.76 \\
\hline Swell & 0.89 & 0.95 & 0.7 & 0.94 \\
\hline OT & 0.62 & 0.97 & 1 & 1 \\
\hline Harm & 0 & 0 & 0.64 & 1 \\
\hline Total & $\mathbf{0 . 6 5 2}$ & $\mathbf{0 . 7 1 7}$ & $\mathbf{0 . 7 9 5}$ & $\mathbf{0 . 8 8 5}$ \\
\hline
\end{tabular}

Table 7. Classification results using ST based pattern and PNN

The obtained results using ST based pattern are hardly better with BP than PNN. As can be observed the classification results are quite robust against the noise using BP. For disturbances the worst results are obtained for sag and interruptions.

Anyway, the results using ST based pattern and BP are better than WT based pattern and any ANN used, moreover the ST based pattern no needs additional features, as occurs with WT based pattern. On the other hand, the total number of operations to compute ST is greater than WT.

\section{Checking the resulting classification systems}

In this section, a data set of power quality disturbances has been generated using the power network simulation environment PSCAD/EMTDC (PSCAD, 2005). This application is an industry standard simulation tool for studying the transient behaviour of electrical networks.

The aim is to obtain different power quality disturbances of data set used for training and validation, in order to check the implemented system.

Several electrical systems with different events, typical and simple, as faults, switching big loads, non linear loads, etc., have been simulated to obtain different power quality disturbances. Moreover different signals can be obtained from a single system by using a facility of software simulation.

One example consists of a low voltage load fed by two parallel lines through a power transformer as shown in Fig. 6. A three phase fault occurs in the middle of one of the two 
parallel transmission lines at a given time. Approximately two cycles after $(t=0.045 \mathrm{~s})$ the fault is cleared. In Fig. 7 (a) can be seen the signal generated, where it can be observed an oscillatory transient produced by the fault, voltage sag during two cycles, and other oscillatory transient produced when the fault is cleared.

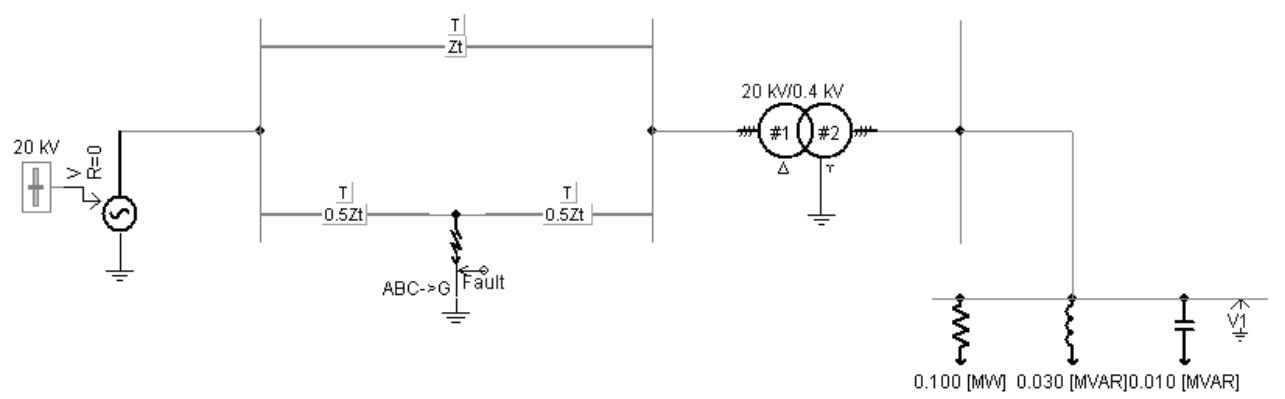

Fig. 6.3 bus test system scheme in PSCAD

The signals shown in Fig. 7 (b)-(c) belong to initial data set used for training stage of classifier systems. In Fig. 7 (b) it is shown a normal voltage to 0.9 , just in the boundary with voltage sag. Fig. 7 (c) depicted a very light oscillatory transient and, just after, a slightly magnitude diminution.

The values of the features in WT based pattern for signals of Fig. 7 (a)-(c) are shown in Table 8 , in rows $\mathrm{A}$ to $\mathrm{C}$, respectively. As can be observed the signal $\mathrm{A}$ (simulation) present a higher value of energy in relative high frequencies D1 $\left(32 \mathrm{f}_{1} \div 16 \mathrm{f}_{1}\right)$ than $\mathrm{C}$, therefore $A$ has an oscillatory transient. The minimum of RMS value for A, lower than B, gives information about the diminution of the magnitude, voltage sag.
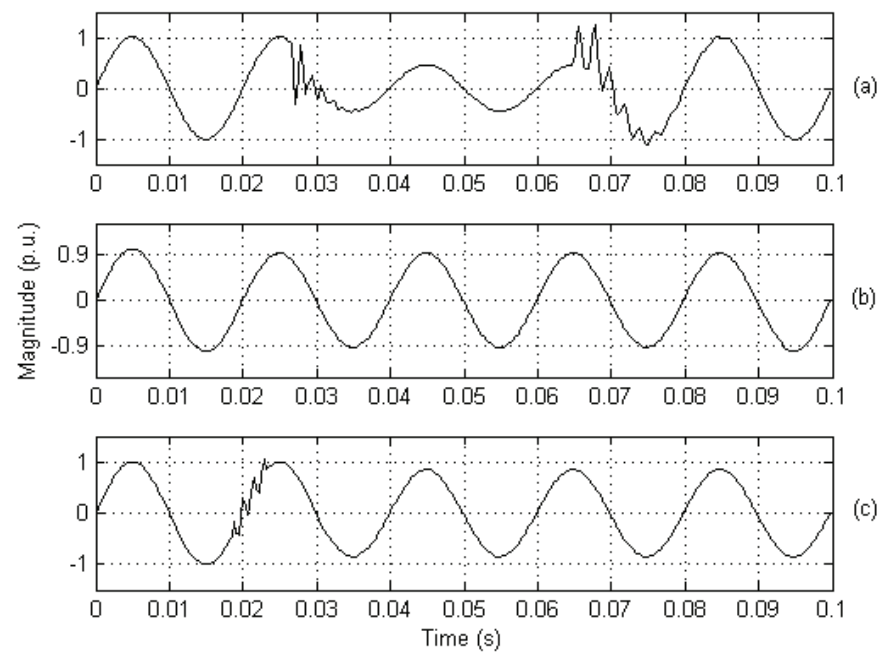

Fig. 7. Signal generated by model simulation (a). Normal voltage to 0.9 (b). Oscillatory transient and a slightly magnitude diminution. 
From Table 8 the obtained values of the features for these signals, any efficient classifier can properly assign the disturbance to each corresponding class.

\begin{tabular}{|c|c|c|c|c|c|c|c|}
\hline & \multicolumn{5}{|c|}{ Energy } & \multicolumn{2}{c|}{ RMS } \\
\hline Signals & D1 & D2 & D3 & D4 & D5 & Max & Min \\
\hline A & 407.9 & 336.6 & 3.164 & 0.920 & 0.675 & 1.043 & 0.457 \\
\hline B & 2.426 & 1.281 & 0.959 & 0.885 & 0.897 & 1 & 0.9 \\
\hline C & 351.4 & 27.22 & 0.722 & 0.794 & 0.801 & 1.012 & 0.849 \\
\hline
\end{tabular}

Table 8. Feature values of WT based pattern for different signals

In order to compare the ST based pattern for these same signals are shown several ST contours. In Fig. 8 (a) it is shown the signal from simulation. The fundamental frequency ST contour for this signal and the normal one to 0.9 is showed in (b). The $700 \mathrm{~Hz}$ ST contour of the simulation signal and the oscillatory transient is depicted in (c). It can be observed that the simulation signal contours advise the presence of voltage sag, the $50 \mathrm{~Hz}$ ST contour for simulation signal (A) is below normal voltage to 0.9 (B), moreover an oscillatory transient because the energy in $700 \mathrm{~Hz}$ ST contour for simulation signals (A) is higher than oscillatory transient (C) used as reference. These graphs show that any efficient classifier can properly allocate the disorder to each corresponding class.

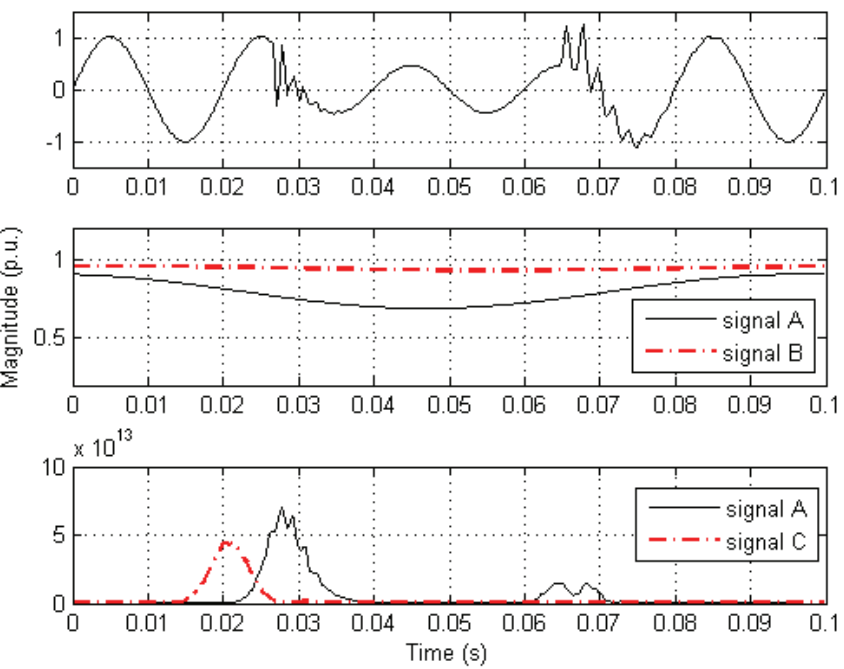

Fig. 8 . Signal generated from simulation (a), comparison of $50 \mathrm{~Hz}$ ST contours (b) and comparison of $700 \mathrm{~Hz}$ ST contours (c)

Others systems have been simulated using PSCAD and the power quality disturbances obtained are showed in Table 9, which also indicates the classification results using resulting classification systems.

These signals verify the behaviour of the implemented system with power quality disturbances based on electrical models, and therefore close to real ones. 


\begin{tabular}{|c|c|c|c|c|}
\hline & & \multicolumn{3}{|c|}{ pattern-ANN } \\
\hline Disturbance & Number & WT-BP & WT-PNN & ST-BP \\
\hline Normal & 10 & 0.90 & 0.90 & 1 \\
\hline Sag & 40 & 0.875 & 0.80 & 0.9 \\
\hline Int & 10 & 0.80 & 0.70 & 0.9 \\
\hline Swell & 30 & 0.933 & 0.866 & 0.933 \\
\hline OT & 30 & 0.866 & 0.933 & 0.933 \\
\hline Harm & 30 & 0.933 & 0.933 & 0.933 \\
\hline Total & 150 & 0.893 & 0.866 & 0.926 \\
\hline
\end{tabular}

Table 9. Classification results for signals obtained by simulation software

As can be observed the results with signals obtained by electrical networks simulation are worse than those obtained with generated following mathematical models. The BP results do not worsen than PNN. At last the classification system using ST based pattern are better than WT based pattern.

\section{Conclusion}

Two different automated classification systems has been implemented, based on timefrequency transforms, WT and ST, in combination with Artificial Neural Network as algorithm classifier.

The classification results are very similar using two different algorithm classifiers, a BP and PNN, so it can be affirmed that is more important designing a properly and suitable pattern that the election of the algorithm. Any efficient classifier algorithm can do its expected job. Therefore the extraction and selection features both are very crucial to the effectiveness of classification applications. Suitable features can greatly decrease the workload and simplify the subsequent process. Generally, ideal features should be of use in distinguishing patterns belonging to different class, immune to noise, easy to extract and interpret.

It can be appreciate that the detail signals of Wavelet transform analysis are very similar in the different disturbances, and in some cases almost identical, therefore becomes necessary to add other features, RMS value, which can distinguish magnitude disturbances. The Stransform generates several contours that put in evidence the nature of the disturbance, and these contours have certain characteristics that are suitable for automated pattern recognition.

On the other hand, the computational cost for Wavelet transform is less that for S-transform, $N$ and $N \cdot(N+\log N)$ respectively.

The implemented systems have been tested with signals generated with simulation program obtaining similar results.

A further limitation of many of the studies is that both training and testing are based in signals obtained following mathematical models. In this paper signals generated by power network simulation have been used to verify the classification system. In any case it becomes necessary to have power quality disturbances available to all researchers in this field that can be reliably used to compare classification results of each approach and not every researcher uses their own signals, whether synthetic, simulated or acquired.

The implemented system has been tested with signals generated with simulation program obtaining very good results. 


\section{References}

Borras, D.; Castilla, M. Moreno, N. \& Montano, J.C. (2001) Wavelet and neural structure: a new tool for diagnostic of power system disturbances. IEEE on Industry Applications, Vol. 37 (Jan. 2001) pp. 184-190

Burrus, C. S.; Gopinath, R. A.; Guo, H. (1998). Introduction to wavelets and wavelet transforms, Prentice Hall, ISBN: 0134896009, New Jersey, USA

Cybenko, G. (1989). Approximation by superpositions of a sigmoidal function. Mathematics of Control, Signals and Systems, No. 2 (1989) pp. 303-314

Dash, P.K.; Panigrahi, B.K. \& Panda; G. (2003). Power quality analysis using S-transform. IEEE Trans. on Power Delivery, Vol. 18, (Apr. 2003) pp. 406-411

Dugan, R.S.; McGranaghan, M.F.; Santoso, S. \& Beaty, H.W. (2000). Electrical Power System Quality, Wiley-Intersciencie, ISBN 007138622X, New York, USA

Gargoom, A.M.; Ertugrul, N. \& Soong, W.L. Automatic classification and characterization of power quality events. IEEE Trans. on Power Delivery, Vol. 23, (Oct. 2008) pp. 24172425

Hecht-Nielsen, R. Counterpropagation networks. Applied Optics, No. 26 (Oct. 1989) pp. 49794984

Mallat, S. (1999). A Wavelet Tour of Signals Processing, Academic Press, ISBN 0123743702

MATLAB (2000). Natick, MA: Math Works, Inc., 2000.

Mishra, S.; Bhende, C.N. \& Panigrahi, B.K. (2008). Detection and classification of power quality disturbances using S-transform and probabilistic neural network. IEEE Trans. on Power Delivery, (Jan. 2008)Vol. 23.

PSCAD (2005). Manitoba HVDC Research Centre, Inc., 2005.

Santoso, S.; Powers, E.J. \& Grady, W.M. (1994). Electric power quality disturbance detection using wavelet transform analysis, Proceedings of IEEE - SP International Symposium on Time- Frequency and Time Scale Analysis, pp. 166-169

Specht, D.F. Probabilistic neural networks. Neural Network, (1990) Vol. 3, pp. 109-118

Stockwell, R.G.; Mansinha, L. \& Lowe, R.P. (1996). Localization of the complex Spectrum: the S-transform. IEEE Trans. on Signal Processing, No. 44, (1996) pp. 998-1001

Widrow, B.; Rumelhard, D.E. \& Lehr, M.A. (1994) Neural networks: Applications in industry, business and science. Commun. ACM, Vol. 37, pp. 93-105

$\mathrm{Xu}, \mathrm{R}$. \& Wunsch D. (2005). Survey of Clustering Algorithms. IEEE Trans. on Neural Networks. Vol. 16, No. 3 (May. 2005) pp. 645-678

Zhang, G.P. (2000).Neural Networks for Classification: A Survey. IEEE Trans. on Systems, Man, and Cybernetics - part c: applications and reviews. Vol. 30, No. 4, (Nov. 2000) pp. 451-462 


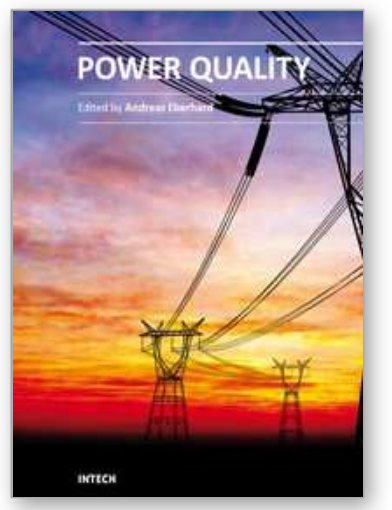

\author{
Power Quality \\ Edited by Mr Andreas Eberhard
}

ISBN 978-953-307-180-0

Hard cover, 362 pages

Publisher InTech

Published online 11, April, 2011

Published in print edition April, 2011

Almost all experts are in agreement - although we will see an improvement in metering and control of the power flow, Power Quality will suffer. This book will give an overview of how power quality might impact our lives today and tomorrow, introduce new ways to monitor power quality and inform us about interesting possibilities to mitigate power quality problems.

\title{
How to reference
}

In order to correctly reference this scholarly work, feel free to copy and paste the following:

Alejandro Rodríguez, Jose A. Aguado, Jose J. López, Francisco Martín, Francisco Muñoz and Jose E. Ruiz (2011). Time-Frequency Transforms for Classification of Power Quality Disturbances, Power Quality, Mr Andreas Eberhard (Ed.), ISBN: 978-953-307-180-0, InTech, Available from:

http://www.intechopen.com/books/power-quality/time-frequency-transforms-for-classification-of-power-qualitydisturbances

\section{INTECH}

open science | open minds

\section{InTech Europe}

University Campus STeP Ri

Slavka Krautzeka 83/A

51000 Rijeka, Croatia

Phone: +385 (51) 770447

Fax: +385 (51) 686166

www.intechopen.com

\section{InTech China}

Unit 405, Office Block, Hotel Equatorial Shanghai

No.65, Yan An Road (West), Shanghai, 200040, China

中国上海市延安西路65号上海国际贵都大饭店办公楼405单元

Phone: +86-21-62489820

Fax: +86-21-62489821 
(C) 2011 The Author(s). Licensee IntechOpen. This chapter is distributed under the terms of the Creative Commons Attribution-NonCommercialShareAlike-3.0 License, which permits use, distribution and reproduction for non-commercial purposes, provided the original is properly cited and derivative works building on this content are distributed under the same license. 